\title{
The knowns \& unknowns of pulmonary toxicity following immune checkpoint inhibitor therapies: a narrative review
}

\author{
Alistair R. Miller ${ }^{1,2,3}$, Renee Manser ${ }^{1,2,4}$ \\ ${ }^{1}$ Department of Respiratory and Sleep Medicine, Royal Melbourne Hospital, Victoria, Australia; ${ }^{2}$ Department of Internal Medicine, Peter \\ MacCallum Cancer Centre, Victoria, Australia; ${ }^{3}$ Department of Medicine, Monash Health, Monash University, Victoria, Australia; ${ }^{4}$ Department of \\ Medicine, Royal Melbourne Hospital, The University of Melbourne, Victoria, Australia \\ Contributions: (I) Conception and design: Both authors; (II) Administrative support: Both authors; (III) Provision of study materials or patients: \\ Both authors; (IV) Collection and assembly of data: Both authors; (V) Data analysis and interpretation: Both authors; (VI) Manuscript writing: Both \\ authors; (VII) Final approval of manuscript: Both authors. \\ Correspondence to: Dr. Alistair R. Miller. Department of Respiratory and Sleep Medicine, Royal Melbourne Hospital, 300 Grattan St., Parkville, \\ Victoria, 3050, Australia. Email: Alistair.miller3@mh.org.au.
}

\begin{abstract}
Since their discovery immune checkpoint inhibitors (ICI) have dramatically changed the treatment landscape for many cancers. In addition to their efficacy they are generally well tolerated, however, they have led to a new range of immune-related adverse events (irAEs) including pneumonitis. While not the most frequently reported immune-related adverse event in the clinical trial setting, recent real-world data suggests a significantly higher rate of pneumonitis leading to treatment suspension or cessation. It also appears to disproportionately contribute to immune-related mortality, particularly with anti-PD-1/PDL1 treatment. While indicators have emerged regarding risk factors, incomplete prospective recording of patient characteristics hampers strong conclusions. Presenting symptoms are non-specific and the differential diagnosis is broad, made more complex by concomitant treatment with traditional chemotherapy or radiotherapy. Radiological findings are diverse and inconsistent terminology makes comparison and more complete characterization difficult. Further, little is known about the role of baseline testing or surveillance for early detection of pneumonitis, or the real-world role of bronchoscopy or biopsy in assessment. Scant literature exists to direct these complex decisions, so treatment guidelines have been published based on expert consensus. Here we provide a narrative review of what is known about ICI pneumonitis and propose key questions to enhance our understanding into the future.
\end{abstract}

Keywords: Pneumonitis; checkpoint inhibitors; immune-related adverse events

Submitted Jul 03, 2020. Accepted for publication Nov 05, 2020.

doi: $10.21037 /$ tlcr-20-806

View this article at: http://dx.doi.org/10.21037/tlcr-20-806

\section{Introduction}

In 2010 a landmark randomized controlled trial was published demonstrating a significant improvement in overall survival in patients with metastatic melanoma treated with ipilimumab. This monoclonal antibody potentiated an antitumor T-cell response by targeting cytotoxic T-lymphocyte-associated antigen 4 (CTLA-4) (1). In the decade since, the extraordinary potential of immune checkpoint inhibitor (ICI) therapy has been further realized, transforming the management of many metastatic malignancies including non-small cell lung cancer (NSCLC) $(2,3)$. ICI has an expanding role in the management of earlier stage disease and is currently being used as consolidative therapy in unresectable stage III NSCLC treated with combination chemotherapy and radiation (4) and ongoing clinical trials are exploring the potential role in neoadjuvant therapy for resectable NSCLC (5). In addition, combination therapy with chemotherapy as first line therapy is under investigation, including in the PEARL 
study (ClinicalTrials.gov MCT03003962), which has great promise, but may also add to the differential for pulmonary infiltrates.

Immunotherapy is not a new concept, with records as early as the work of Coley in 1890 detailing attempts to modify or harness a host's own immune response in fighting cancer (6). Modern immunotherapy encompasses a wide variety of treatments including vaccines and adoptive cell transfer. However, the recent resurgence of immune based therapies is largely thanks to the discovery of the mechanism by which cancer cells evade the T-cell mediated antitumor immunity, that is by dysregulating the expression of immune checkpoint proteins (7). There are a great many co-stimulatory and inhibitory molecules that play a role in T-cell regulation, but the seminal work of Honjo and Allison enabled a focus on the programmed cell death (PD1)/PD-Ligand 1 and CTLA-4 pathways respectively $(8,9)$. These two T-cell protein receptors, and their ligands, are elements of the inhibitory pathways of the immune system critical for sustaining self-tolerance and tempering the impact of physiological immune responses to limit collateral tissue damage (7).

Tumor-induced immune tolerance can be overcome and $\mathrm{T}$ cell response to tumors enhanced by blocking the ligand-receptor interactions responsible for activation of immune checkpoints in several way: (I) antibodies which target PD-1 (pembrolizumab and nivolumab) preventing interaction with its ligands, PD-L1 and PD-L2 (expressed by tumor cells and infiltrating immune cells) $(2,3,10)$; (II) antibodies directly targeting PD-L1 (atezolizumab, avelumab and durvalumab) $(4,11,12)$; (III) anti-CTLA-4 antibodies (ipilimumab and tremelimumab) prevent binding of the associated ligand, CD80 and CD86 $(1,13)$. While both signaling pairs have a role in the inhibition of T-cell activation, they act at different points, with CTLA-4 acting early in the proliferation of T-cells, and the PD-1/PDL1 pairing acting further down the process, on T-cells in peripheral tissues $(14,15)$. This underlies the rationale for using them in combination, with evidence of both additive and synergistic effects of this concurrent use (16), and indeed may explain their differential effects and toxicities.

While the risk-benefit profile of ICI is generally favorable, this unique mechanism of action has given rise to an unparalleled range of autoimmune like side effects termed immune-related adverse events (irAEs) largely due to activation of $\mathrm{T}$ immune cells in a diverse range of tissues (17). The most common pulmonary adverse event is pneumonitis with an overall incidence of $2.7 \%$ in clinical trials of PD-1 inhibitors (18), although as we will discuss, real-world data suggests it may be higher outside the trial setting.

This narrative review provides a background to ICI-pneumonitis, discussing what is known about the pathophysiology, epidemiology, risk factors and presentation of pneumonitis, presents current recommendations for treatment, and finishes by proposing key questions to extend our understanding of this condition. We present the following article in accordance with the Narrative Review reporting checklist (available at http://dx.doi.org/10.21037/ tlcr-20-806).

\section{Methods}

We identified key published articles and conference abstracts using the PubMed search engine and Google Scholar, published in English without time limitations up to June 2020. The search was conducted using the terms 'checkpoint inhibitors', 'immunotherapy' and 'pneumonitis' regardless of the primary tumor type. Secondary review of bibliographies of key meta-analyses was also performed to ensure all important literature was included.

\section{Terminology}

Inconsistencies in terminology used to describe pulmonary irAEs were highlighted recently by a multidisciplinary international panel in a research statement produced by the American Thoracic Society (ATS) (19). While the term ICI-interstitial lung disease (ILD) has been suggested by some investigators, the ATS panel has recommended the using the term ICI-related pneumonitis (ICIP) $(19,20)$. The panel have recommended avoiding terms such as pulmonary toxicity which may imply a dose-response relationship. The common terminology criteria for adverse events (CTCAE) defines pneumonitis as "A disorder characterized by inflammation focally or diffusely affecting the lung parenchyma" (CTCAE version 5.0). The term pneumonitis is generally used to describe inflammation that is noninfectious but lacks a clear and widely accepted specific definition.

\section{Pathophysiology of pneumonitis}

Like other irAEs, the pathophysiology of pneumonitis is incompletely understood $(14,17)$. As mentioned above, the checkpoint proteins play a crucial role in self-tolerance, 
so it is not surprising that inhibition leads to a degree of autoimmunity. It is both plausible and likely that there is some cross-reactivity of tumor antigens with other normal host tissues. Consistent with this, in both myositis and skinrelated AEs, identical T-cell clones have been identified in tumor tissue and the site of irAEs (17). Certainly, bronchoalveolar lavage (BAL) in pneumonitis has shown a lymphocyte predominance (20-22) and Suresh et al. found a significant increase in central memory T-cells in BAL of patients with ICIP (23). However, dysregulated cellular immunity is not the only proposed mechanism. Interrogation of the WHO global pharmacovigilance database has shown the cytokine release syndrome may occur in response to ICI (24), although is not as common as with CAR $\mathrm{T}$ cell therapy (25). Lim et al. showed that a panel of circulating cytokines predicted severe irAEs, including pneumonitis (26). In ICI-related colitis, elevated IL-17 has been proposed as a causative mechanism (27), raising the possibility of cytokine driven disease elsewhere. A case report by Naqash et al. describe the successful treatment of ICI-related pneumonitis with tocilizumab, an anti-IL6 antibody (28), adding to a picture of dysregulated inflammatory pathways.

Whilst the anti-tumor effect of ICIs is predominantly through changes in T-cell signaling, there is a recognized effect on B-cells. In a study by Das et al. the number of circulating B cells was significantly reduced in patients treated with dual-ICIs, and these changed were associated with higher rates of irAEs (29). In both thyroid and skinrelated AEs, specific auto-antibodies have been identified, although it is unclear whether these were present prior to the ICI therapy $(14,17)$, and whether such antibodies exist in the context of pneumonitis. Much remains unknown, and thus specific and targeted preventative and treatment strategies remain elusive.

\section{Epidemiology of ICI pneumonitis}

Early studies of immunotherapy described the new range of adverse effects that were distinct from traditional cytotoxic chemotherapy (30). These included diverse immunerelated effects but were most commonly manifested as dermatologic, gastrointestinal and endocrine disorders $(1,10)$. Treatment for melanoma was first described with studies reporting dyspnea but no significant rate of pneumotoxicity (1). However, as further experience was gained with this new therapy case reports $(10,31)$ and metaanalyses showed not only that pneumonitis occurred but that it contributed significantly to treatment related death (32). In a 2015 meta-analysis examining 11 studies, the incidence of pneumonitis ranged between $1.3 \%$ and $11 \%$, with no clear signal as to differences between agents (ipilimumab, nivolumab or pembrolizumab) or malignancy (melanoma, castrate-resistant prostate cancer or NSCLC) (32). The majority of the pneumonitis was mild (grade 1-2) with reports of complete resolution with immune suppression, usually using glucocorticoids, and only one reported death.

In a more contemporary meta-analysis, $\mathrm{Su}$ et al. report on data from nearly 13,000 subjects across 23 randomized controlled trials, including 8 of the trials reported in the 2015 analysis (33). The analysis included studies using the PD-1 inhibitors nivolumab and pembrolizumab, the PD-L1 inhibitors durvalumab and atezolizumab, and the CTLA-4 inhibitors ipilimumab and tremelimumab, looking at rates on ICIP and pneumonia. They showed an increase in any grade pneumonitis for both PD-1 and PD-L1 inhibitors, with a RR 5.17 and 3.25, respectively. However, only PD-1 inhibitors, particularly pembrolizumab, showed an increase in higher-grade [3-5] pneumonitis (RR 5.64). In contrast, they did not find an increased risk in those receiving CTLA-4 inhibitors. The pattern of risk was not significantly different in NSCLC or other tumors. No agents or groups were associated with an increase in pneumonitis-related death.

Notably, in advanced melanoma, and in later in lung cancer, dual treatment with ipilimumab and nivolumab has resulted in higher rates of pneumonitis. In the Checkmate 067 trial in advanced melanoma, pneumonitis was nearly 4 fold higher in the dual-therapy group than that of the single treatment arms (34), although the rates of highgrade pneumonitis were not different. These results were confirmed in the study by Postow et al. examining the same regimen (35). In a study comparing ipilimumab-nivolumab to single agent nivolumab or chemotherapy in advanced non-small cell lung cancer, pulmonary adverse events were twice as common in the ipilimumab-nivolumab group as compared to the group receiving only nivolumab. In addition, pneumonitis contributed disproportionately to death, with 4 of 8 deaths attributed to pneumonitis, despite pneumonitis only making up around $10 \%$ of all adverse events (36). This is also reflected in non-trial populations, as described by Suresh et al. in their 2018 paper describing 205 subjects on ICI therapy both in, and outside, trial (37). Interestingly, they also showed a significantly higher incidence of ICI pneumonitis than that seen in clinical trials to that point, with a rate of $19 \%$ adjudicated by a 
multi-disciplinary team. Similarly, in a recent populationbased study from an administrative dataset in the US, the incidence of pneumonitis was $14.3 \%$ at 9 months after treatment (38) and an incidence of $8.8 \%$ was reported in a multicenter retrospective review from Japan (39). Of greater concern, are the rates of severe (grade 3-4) ICIP reported in real-world studies with a prevalence as high as $5.7 \%$ (40). Wang et al. interrogated the WHO pharmacovigilance database of irAEs, describing the nature and range of immune related fatal events (41). They showed significant variation in the predominant manifestation between regimens, with fatal events more commonly related to ICIP when treated with anti-PD-1/PD-L1 agents, while colitis was the primary cause of death related to ipilimumab (the sole CTLA-4 agent). Interestingly, when expressed as a percentage of all irAEs, fatal events accounted for approximately $14 \%$ of all reported pneumonitis, as compared with colitis and endocrine disorders $(<5 \%)$. There was also a clear reduction in the time to onset of symptoms in the combination therapy group as compared to any single agent therapy, 14.5 vs. 40 days (41). In a research letter, Moey et al. also examine the $\mathrm{WHO}$ pharmacovigilance database showing a shorter time to onset for fatal cases, however, while there has been an overall increase in report of ICI pneumonitis the rate of fatal disease has been stable (42).

\section{Risk factors}

As mentioned above, there appears to be class and agent specific variation in the risk of developing ICIP (43-46), with anti-PD-1 and PD-L1 agents, and combination therapy, having a higher rate of pneumonitis than single agent anti-CTLA-4 treatment. Beyond this, several other risk factors have been suggested, although none at this point have adequate data to constitute a contraindication to therapy. There does appear to be some signal related to an increased risk with smoking, although no studies have shown a definitive difference (45), perhaps because current and former smokers are grouped together rather than separately.

The presence of ILD has been shown in a number of settings to increase the risk of ICIP $(47,48)$. In a retrospective review of patients receiving nivolumab across three Japanese hospitals, Kanai et al. showed that those with pre-existing ILD had equivalent survival but more than twice the rate of ICIP, $31 \% v s .12 \%$. Interestingly the ILD group had a higher percentage of current or former smokers, and none had an EGFR or ALK mutation, as opposed to $25 \%$ in the non-ILD group (48). Similarly, pre-existing lung disease was associated with higher rates of pneumonitis in a retrospective case-control study of 55 patients in China (45). Cho et al. have provided further insights to the risk of different chronic lung disease, showing in a Korean population with NSCLC that ILD, but not COPD or bronchiectasis, increased the risk of ICIP (47). However, it is likely that the severity of the underlying lung disease is important in the degree of risk. Fujimoto et al. showed safety of nivolumab in the treatment of NSCLC in a small cohort of patients with very mild ILD (49). In their cohort of 18 patients, there were no treatment related deaths, and only 2 patients had mild (grade 2) pneumonitis.

Whether radiotherapy increases the risk of ICI pneumonitis remains somewhat contentious, not in the least because differentiation between radiation pneumonitis and ICIP is challenging. In the KEYNOTE-001 trial, prior thoracic radiation was associated with an increased risk of pneumonitis $(13 \%$ vs. $1 \%)$ in those treated with pembrolizumab (50). Similarly, in the PACIFIC study all grades of pneumonitis or radiation pneumonitis were more common in patients with stage III unresectable NSCLC treated with durvalumab post concurrent chemoradiation (33.9\%) compared with those treated with placebo post chemoradiation $(24.8 \%)$ (51). The incidence of grade 3 and 4 pneumonitis or radiation pneumonitis were similar between the 2 groups (3.4\% and $2.6 \%$, respectively), however the proportion with grade 2 pneumonitis was not described. Three recent observational studies report rates of pneumonitis in the real-world setting. A low rate of pneumonitis of $1.1 \%$ was reported by Avrillon et al. in a French study of 591 participants receiving Durvalumab post definitive chemoradiotherapy (52). Miura et al. reported that $61 \%(25 / 41)$ developed pneumonitis in the setting of Durvalumab treatment post chemoradiation for stage III NSCLC in a cohort studied at the Saitama Medical University, International Medical Center in Japan (53). In more than $50 \%$ of the cohort durvalumab was ceased due to pneumonitis. Six patients were rechallenged, with 2 subsequently developing an exacerbation of pneumonitis. The relatively high rate of pneumonitis in this study was comparable to that seen in the subset of Japanese participants in the PACIFIC trial (53). In another single institution study from the Samsung Medical Center, Republic of Korea, a relatively high rate of grade 3 pneumonitis was reported (14.3\%) (54). Of note, however subgroup analysis in the PACIFIC trial showed that rates of pneumonitis were higher amongst Asian participants 
and this was not increased in those receiving durvalumab compared with placebo (55) suggesting perhaps a racial disparity in risk for the development of pneumonitis.

Like other systemic therapies $(56,57)$, ICIs have been associated with radiation recall pneumonitis (RRP) (58). In a study of real-world outcomes in patients treated with Nivolumab in Japan, RRP was reported in approximately $5 \%$ of patients who had a history of thoracic radiation, however it appeared to have a better outcome than de novo ICIP (39). Similarly, in a study of nivolumab for second-line or later treatment for NSCLC, ICIP was more common in those with prior radiation pneumonitis $(36.5 \%$ vs. $9.6 \%)$ but was associated with a better progression free survival (59).

Given the known differences in immune response between sexes (60), several groups have investigated whether this manifests as differences in efficacy and tolerability of ICIs. In a large systematic review, Conforti et al. showed that while ICIs had beneficial effects in men and women, the magnitude of the benefit was greater in men (61), although whether this is confounded by other factors including smoking rates has been argued (62). Duma et al. have shown that women treated with ICIs for NSCLC are more likely to develop irAEs, including pneumonitis (63). All grades of pneumonitis were seen in $11 \%$ of women and $4 \%$ of men, although there were no differences in grades $\geq 3$.

\section{Clinical features}

In those with symptomatic ICIP dyspnea and cough are the most common presenting symptoms, fever is less common (occurring in up to a third) and chest pain relatively infrequent $(20,64)$. A third or more of cases may be asymptomatic at the time of diagnosis, having been detected on routine surveillance imaging $(64,65)$. The majority of cases occur within the first 6 months of treatment with a median time to onset reported between 2.3 and 2.8 months in several studies $(20,37,64)$. Late onset, up to 2 years, is described, however higher grades tend to present earlier $(20,37)$. A tendency to earlier onset has been described in NSCLC (20) and with combination therapy (64).

Differential diagnoses in those presenting with suspected ICIP include infection, disease progression, pseudoprogression (66), pulmonary embolism, pulmonary edema/ myocarditis (67) and respiratory failure due to ICI related neuromuscular disease (68). Sarcoidosis like granulomatous disease has been associated with ICI therapy $(65,69)$ and reactivation of TB has also been reported (70). In addition, there are emerging case reports of obstructive bronchiolitis/ airways disease associated with ICI therapy (71-73). An increase in the risk of pneumonia has been reported in a recent systematic review associated with the use of PD-L1 inhibitors, but not in association with PD-1 and CTLA-4 inhibitors (33).

High resolution computed tomography (CT) scanning is critical in the evaluation of suspected ICIP, however there are no distinct pathognomonic features. The most common findings are ground glass opacities (GGOs) and areas of consolidation $(20,37,64,74,75)$. In their research statement on ICIP the ATS panel has recommended reporting radiological patterns such as cryptogenic organizing pneumonia (COP) pattern, hypersensitivity pattern (HP), non-specific interstitial pneumonitis (NSIP) pattern or acute interstitial pneumonitis (AIP)/acute respiratory distress syndrome (ARDS) pattern should be used to report CT scans rather than characteristic findings. Several groups have used the ATS/ERS international multidisciplinary classification of interstitial pneumonias (76) in retrospective observational studies $(20,64,74,75,77)$.

Across these studies in a variety of settings, the radiological patterns were reported by radiologists, pulmonologists, or oncologists with experience in interstitial pneumonias, generally blinded to clinical details (Table 1). Overall, the commonest pattern was COP, followed by HP and NSIP. However, a significant proportion in 3 of the 5 studies did not fit into one of the interstitial pneumonia patterns, with GGO, unclassifiable interstitial changes and nodular changes described. A change in radiographic subtype over time was observed infrequently (64), but particularly in the study by Delaunay et al. a mixed pattern was described, with asymmetrical topography observed in $20 \%$ of cases. The number of lobes involved varied between 1 and 5 with a median of 3 (20). Baba et al. described one or more atypical features, including some with peri-tumoral ground glass opacities and some with opacities confined to the lung ipsilateral to the tumor (74). This serves to highlight the challenges of classifying ICIP using existing classifications (76). The pathophysiological mechanisms in ICIP may differ to those seen in other ILD and other forms of drug induced ILD (DILD). It is not clear whether the unclassifiable radiological findings observed in some participants in these studies represent a new clinical entity or variants of recognized forms of ILD. There is also limited data about whether particular radiological patterns are associated with overall survival or with ICI-related mortality. Watanabe et al. reported that patients with GGO had significantly worse overall survival compared with those 
Table 1 Reported radiological features of ICIP

\begin{tabular}{|c|c|c|c|c|c|c|c|}
\hline & $\mathrm{COP}$ & NSIP & $\mathrm{HP}$ & AIP/ ARDS & Combination & Other & Unclassified \\
\hline Naidoo et al. (64) & $19 \%$ & & $22 \%$ & & & $37 \%$ GGO, $7 \%$ interstitial change & $15 \%$ NOS \\
\hline Delauney et al. (20) & $24 \%$ & $8 \%$ & $16 \%$ & & $10 \%$ COP and NSIP & $6 \%$ bronchiolitis & $36 \%$ NOS \\
\hline Baba et al. (74) & $47 \%$ & $8 \%$ & $24 \%$ & $13 \%$ & & & $7 \%$ \\
\hline
\end{tabular}

COP, cryptogenic organising pneumonia pattern; NSIP, non-specific interstitial pneumonitis pattern, HP, hypersensitivity pattern; AIP/ ARDS, acute interstitial pneumonitis/acute respiratory distress syndrome pattern; GGO, ground glass opacity; NOS, not otherwise specified.

with COP (75), while Baba et al. reported that 68\% (13/19) of those with an AIP/DAD pattern died in association with ILD (74). Of course, these findings are not exclusive, and several other radiological findings have also been described. Adding to confusion around differential diagnosis, hilar and mediastinal lymph nodes have been noted to be enlarged in the context of pneumonitis or granulomatous disease. Airways may also be involved in immune-related pulmonary disease, with the development of areas of ground-glass opacity surrounded by denser tissue, or a reverse-halo sign (78).

Bronchoscopy with BAL plus or minus transbronchial biopsy (TBB) is suggested in the work up of suspected grade 2 ICIP and recommended in the evaluation of grade 3 and 4 ICIP according to current guidelines (79). While BAL and biopsy results may provide ancillary diagnostic information, there are no cytopathological features which are pathognomonic for ICIP. As with other types of DILD, ICIP is primarily a diagnosis of exclusion $(80,81)$. The most important role of bronchoscopy is to exclude other causes such as infection or disease progression (e.g., lymphangitis). Delaunay et al. reported the largest series of BAL cell count results in ICIP. BAL was performed in 35 out of 64 participants and differential cell count was available in 30 , $80 \%$ of which showed a lymphocytosis of $>15 \%$. Elevated neutrophils and eosinophils were observed in some cases (20). Increased BAL lymphocytosis has also been reported in another small series (23). Baba et al. reported that 15 out 144 patients included their study had BAL performed; the median proportion of lymphocytes was $35.8 \%$ (range: $1.3 \%$ to $76.8 \%$ ) but further details were not provided. The riskbenefit of performing bronchoscopy needs to be carefully considered on an individual basis. Routine infectious work up including sputum, urine, blood cultures and nasal swab are recommended for those with grade 2 and above pneumonitis (79).

Routine lung biopsy is not recommended (82). However, where there is clinical or radiological uncertainty about the etiology of a pulmonary infiltrate, biopsy may be indicated. European Society of Medical Oncology (ESMO) guidelines suggest that video assisted thoracoscopic surgery is more likely than TBB to secure a specific diagnosis. However, the guideline panel acknowledges that TBB has a role in the diagnosis of infection, malignancy and possibly granulomatous disease and organizing pneumonia. They also highlight that any decision to undertake a surgical biopsy will depend on patient risk, the location and distribution of radiological changes and health system factors (82).

In the study by Naidoo et al., 11 participants had undergone biopsy ( 8 transbronchial, 2 core biopsies and one surgical), 4 of which were reported to show cellular interstitial pneumonitis, 3 organizing pneumonia and 1 diffuse alveolar damage, while 3 showed no significant abnormality (64). Poorly formed granulomas were observed within the interstitial infiltrate in 3 cases and eosinophils were observed in 2. One autopsy case report describes histological changes of an established sarcoidlike granulomatous reaction of the lung, pulmonary fibrosis and diffuse alveolar damage in a patient who had been treated with ipilimumab followed by nivolumab and died with metastatic melanoma (83). Shea $\mathrm{et} \mathrm{al}$. reported a case of ICIP which initially presented with COP and progressed rapidly to ARDS which was confirmed histologically at autopsy (84). Delaunay et al. reported that 6 participants in their cohort of 64 had undergone transbronchial biopsy which showed inflammatory and lymphocytic infiltration, but further descriptive information was limited (20).

In a retrospective series of 9 patient with suspected ICIP who had undergone lung biopsy reported by Larsen $e t$ al., 
organizing pneumonia was seen in 7 patients admixed with vague non-necrotizing airspace granulomas in 3 cases. One patient had acute fibrinous pneumonitis and one diffuse alveolar damage. Those with organizing pneumonia pattern had mild or asymptomatic disease, while the other 2 died. All cases showed foamy macrophages and pneumocyte vacuolization and 6 had rare eosinophils (85).

Lung function tests have not been reported in the majority of observational studies of ICIP to date. In the study by Naidoo et al. spirometry $(\mathrm{n}=16)$ and diffusing capacity of lung for carbon monoxide adjusted for hemoglobin $(\mathrm{n}=12)$ were completed in a subset of patients at the time of pneumonitis, however detailed results were not provided. They observed that there was no association between lung function parameters and clinical outcome in this small subgroup (64). In a series of 3 patients Leroy et al. reported restrictive lung function with reduced total lung capacity and arterial oxygen desaturation on exertion in one case and normal lung function tests in another (21).

\section{Early detection and prevention}

Early detection of ICIP has the potential to reduce associated morbidity and mortality. Currently some asymptomatic cases are detected by periodic CT scanning undertaken during routine monitoring of malignant disease $(64,65)$. This type of CT monitoring is usually only undertaken every few months. More frequent testing might be feasible with pulmonary function tests (PFTs). Pulmonary function testing, particularly monitoring of $\mathrm{CO}$ diffusion capacity (DLCO) has been investigated in the setting of chemotherapeutic agents associated with DILD, however the evidence base is limited and there is uncertainty about the sensitivity and specificity of PFTs (86). In particular, asymptomatic falls in DLCO are relatively common in studies which have monitored for DILD (86). Franzen et al. undertook serial monitoring with spirometry and DLCO in 71 patients with metastatic melanoma before and during treatment with ipilimumab (87). A reduction from baseline of forced vital capacity (FVC) of $\geq 10 \%$, or $\geq 15 \%$ of DLCO was defined as clinically meaningful suggesting pulmonary toxicity. A clinically meaningful decline in lung function was observed in $24 \%$ of patients at 9 weeks, however only $7 \%$ reported respiratory symptoms and only one patient was diagnosed with ICIP (87). These results highlight the potential challenges associated with screening. When the performance characteristics of the test are suboptimal and the prevalence of disease is low, many 'positive' test results will be false positives leading to further investigations and possibly unnecessary interruptions to treatment. There are no particular biomarkers currently validated in the setting of ICIP, however in the future biomarkers and/or functional assessments such as 6-minute walk tests might be candidates for early detection. Of course, the ability to accurately predict who will develop irAEs would improve the pick-up rate in screening, and reduce unnecessary testing in those not at risk. Colen et al. report a novel approach to risk stratification, by using baseline radiomic features to predict risk. In a small cohort, they were able to identify radiomic features that retrospectively predicted pneumonitis (88).

A number of early phase trials are currently in progress to investigate whether immunomodulatory agents can be given in conjunction with ICIs to prevent irAEs. Some of the agents being studied include fecal microbiota capsules (for prevention of colitis), certolizumab, infliximab, rituximab and tocilizumab (17).

\section{Treatment}

Several national and international groups have published guidelines on management of irAEs, including for ICIP (79,82,89-91), with three being most frequently referenced: European Society of Medical Oncology (ESMO) published in 2017 (82); American Society of Clinical Oncology (ASCO) published in 2018 (79); and National Comprehensive Cancer Network (NCCN) published in 2019 (91), see Tables 2,3. Whilst considering the published literature, the recommendations are largely consensus based. There is no consistency between the guidelines as to baseline tests, and to a lesser degree investigation at the time of the development of suspected pneumonitis. The guideline published by ASCO does not recommend any specific testing prior to the commencement of therapy, although does document CXR, $\mathrm{CT}$ and respiratory function testing as being commonly performed (79). The NCCN on the other hand suggest measurement of resting and exertional oxygen saturation at baseline and with development of symptoms (91), and suggest consideration of PFTs for 'high-risk' patients, without further definition of this. The ESMO guidelines suggest only imaging at baseline, and to repeat a CT chest as a routine 12 weekly (82).

The escalation of treatment from observation for grade 1 pneumonitis, to outpatient oral corticosteroid treatment for grade 2, to inpatient treatment along with IV corticosteroid for grades 3 and 4 are similar across the guidelines. Monitoring frequency for lower grades of pneumonitis does 
Table 2 Baseline investigation and monitoring

\begin{tabular}{llll}
\hline Variable & ASCO (75) & NCCN (86) & ESMO (78) \\
\hline $\begin{array}{l}\text { Routine baseline } \\
\text { investigation }\end{array}$ & $\begin{array}{l}\text { CXR/CT; spirometry and } \\
\text { diffusing capacity }\end{array}$ & $\begin{array}{l}\text { Oxygen saturation at rest and } \\
\text { ambulatory; PFTs for 'high-risk' patients }\end{array}$ & Baseline CT; repeated every 12 weeks \\
$\begin{array}{ll}\text { Investigation for } \\
\text { pneumonitis }\end{array}$ & $\begin{array}{l}\text { CXR, CT chest; oxygen } \\
\text { saturation; infectious workup } \\
\text { for grade 2 or higher }\end{array}$ & $\begin{array}{l}\text { Oxygen saturation based on symptoms; } \\
\text { CT chest; biopsy for exclusion of other } \\
\text { causes }\end{array}$ & FBC/UEC/LFTs/TFTs/Ca/ESR/CRP \\
\hline
\end{tabular}

Table 3 Treatment recommendations

\begin{tabular}{|c|c|c|c|}
\hline Variable & Grade 1 & Grade 2 & Grade 3 and 4 \\
\hline NCCN (86) & $\begin{array}{l}\text { Consider holding } \mathrm{ICl} \text {; monitor } \\
1-2 \text { weekly clinically and with } \\
\text { oxygen saturation (resting and } \\
\text { amb); consider CT } \\
\text { chest-consider repeat in } \\
4 \text { weeks or if worsening } \\
\text { symptoms; resume } \mathrm{ICI} \text { when } \\
\text { radiologic change improves }\end{array}$ & $\begin{array}{l}\text { Hold ICI until < G1 and off steroid; monitor } \\
\text { every 3-7 d clinically and with oxygen } \\
\text { saturation (resting and amb); pulmonary } \\
\text { consultation; consider infectious workup; } \\
\text { consider bronchoscopy with BAL; consider } \\
\text { CT chest-consider repeat in } 4 \mathrm{w} \text {; consider } \\
\text { empiric antibiotics; prednisolone } \\
1-2 \mathrm{mg} / \mathrm{kg} / \text { day-taper once G1 then taper over } \\
4-6 \text { weeks; if no improvement by } 72 \mathrm{~h} \text { treat } \\
\text { as G3 }\end{array}$ & $\begin{array}{l}\text { Permanently discontinue ICl; } \\
\text { methylprednisolone } 1-2 \mathrm{mg} / \mathrm{kg} / \\
\text { day-wean over > } 6 \text { weeks; pulmonary } \\
\text { and ID consult; infectious workup; } \\
\text { consider PFTs; consider empiric } \\
\text { antibiotics; bronchoscopy with BAL; if } \\
\text { no improvement within } 48 \mathrm{~h} \text { consider } \\
\text { additional immunosuppression with } \\
\text { infliximab, mycophenolate or IVIG }\end{array}$ \\
\hline ESMO (78) & $\begin{array}{l}\text { Baseline CXR and bloods (U/E, } \\
\text { LFT, CRP, Ca, TFT, ESR, CRP); } \\
\text { consider workup for infection; } \\
\text { consider treatment delay; } \\
\text { monitor for symptoms every } \\
\text { 2-3 days }\end{array}$ & $\begin{array}{l}\text { Withhold ICl; repeat baseline tests weekly; } \\
\text { daily monitoring of symptoms; start empiric } \\
\text { antibiotics if suspicion of infection (fever, CRP, } \\
\text { neutrophils); if no suspicion of infection or no } \\
\text { improvement in } 48 \text { h start prednisolone } \\
1 \mathrm{mg} / \mathrm{kg} / \text { day-wean according to symptoms } \\
\text { over at least } 6 \text { weeks; HRCT +/- } \\
\text { bronchoscopy and BAL }\end{array}$ & $\begin{array}{l}\text { Discontinue ICl; methylprednisolone } \\
2-4 \mathrm{mg} / \mathrm{kg} \text { IV-wean as clinically } \\
\text { indicated over at least } 8 \text { weeks; } \\
\text { HRCT and respiratory review; cover } \\
\text { with empiric antibiotics; discuss } \\
\text { escalation and ventilation; if no } \\
\text { improvement within } 48 \mathrm{~h} \text { consider } \\
\text { adding infliximab, or mycophenolate if } \\
\text { concurrent hepatotoxicity }\end{array}$ \\
\hline
\end{tabular}

Grade 1: asymptomatic; $\leq 1$ lobe or $<25 \%$ of parenchyma involved. Grade 2: limiting instrumental ADLs; $\geq 1$ lobe or $25-50 \%$ parenchyma. Grade 3: severe symptoms limiting personal ADLs; >50\% parenchyma involved; oxygen indicated. Grade 4: life threatening; requiring respiratory support.

vary to some degree, with the ESMO guidelines suggesting the shortest intervals (2-3 days for grade 1 and daily for grade 2), NCCN suggesting the longest interval (1-2 weeks for grade 1 and 3-7 days for grade 2), with ASCO falling in the middle. All recommend corticosteroid as first line and that a response is expected within 48 hours, or treatment should be escalated. Similarly, weaning of the corticosteroid is recommended over $\sim 6$ weeks for grade 2 and $\sim 8$ weeks for grades 3 and 4. Bronchoscopy is recommended for grades $\geq 3$, although it is noted that patients are often too unwell to tolerate this.

Whilst corticosteroids are usually effective, a proportion of people will not respond or have only a partial response, and require additional immunosuppression $(37,64)$. One small series suggested that as many as $42 \%$ of those with grades $\geq 3$ required additional immunosuppression (64). 
There are no prospective comparisons of the most appropriate next agent, but infliximab, mycophenolate, cyclophosphamide and IVIG have all been reported. Infliximab has the most literature, with ample case reports and case series showing benefit in some (92-94). In ICIrelated colitis, it has been associated with faster resolution of symptoms than corticosteroid alone (95).

Whether or not to rechallenge with an ICI remains a matter for debate. There are certainly cases of successful rechallenge with the same or different agents $(64,96)$, within and outside class, and similarly cases of spontaneous recurrence of pneumonitis without rechallenge $(64,97)$.

Of note, while there is no prospective data, retrospective reports have suggested no reduction in efficacy of ICIs where immunosuppression is required during the treatment course for management of irAEs (91). In fact, there is some data suggesting of a link between disease response and the occurrence of irAEs, including pneumonitis $(98,99)$.

\section{Unknowns and research priorities}

It is unanimous across the published literature that there remains much for us to learn about irAEs, including pneumonitis, as our current understanding of the clinical, radiological, and pathological features of ICIP is based on limited quality, generally retrospective observational research. Before treatment even starts, there are no head to head trials which clarify whether there are truly differences in the effectiveness of different ICIs or their propensity to cause irAEs, although there is clearly retrospective data to suggest this. We do not have a strong indication of what constitutes a significant risk factor for the development of pneumonitis, and whether this is specific to the choice of agent or malignancy. Moreover, whether any particular risk factor predisposes to higher grade or fatal pneumonitis is unknown. Prospective collection of patient characteristics including smoking status and the presence of chronic lung disease will be essential in establishing this in the real-world. The current grading system for severity may be inadequate in those with pre-existing chronic lung disease, and studies are needed to evaluate the role of pulmonary function testing pre-treatment for risk stratification (particularly in smokers and ex-smokers) and in the assessment of those with suspected ICI pneumonitis $(19,79)$ and its recovery.

The underlying pathophysiology of irAEs remains incompletely understood, with little systematic prospective data to elucidate this and a scarcity of data on cytopathological features. Whether there are specific triggering events, for example infection, in the development of pneumonitis, or how best to distinguish between infective and inflammatory disease remains under evaluated. The exact role of bronchoscopy with BAL and/or biopsy warrants further evaluation (19). Similarly, in the context of concurrent or sequential treatment with radiotherapy, it is uncertain whether there are specific clinical, radiologic, or pathological features that distinguish between radiation pneumonitis and ICI pneumonitis. The optimal dose and duration of corticosteroid for pneumonitis remains to be shown, and much could be gained from standardized reporting of this in clinical trials. In the context of corticosteroid resistance, we remain unsure what the optimal next immunosuppressive agent is. And finally, whilst some groups have shown successful rechallenge after pneumonitis, it is not well defined if there are specific groups for whom it is safe to challenge with the same agent, or only an agent from another class, or for whom the risk of recurrence is too high.

There is an urgent need for prospective, multidisciplinary, multi-institutional translational and clinical trial research to enhance our understanding of the pathophysiology and risk factors for ICI pneumonitis in the real-world setting. The development of universal set of minimum diagnostic criteria for the diagnosis of DILD in general, and specifically for ICIP would be a substantial advancement for research in this field. There is also a need to develop a unified framework and nomenclature to describe the radiological and pathological features of ICIP. As with other forms of ILD, a multidisciplinary process will be critical for diagnosis and classification. This process will need to incorporate mechanisms that can qualify or quantify uncertainty in a standard way when there is discordance between radiological and/or pathological findings and our current understanding of ILD or between clinical, radiological and pathological findings within individual patients.

\section{Acknowledgments}

Funding: None.

\section{Footnote}

Provenance and Peer Review: This article was commissioned by the Guest Editor (Daniel Steinfort) for the series "Lung cancer and the immune system" published in Translational Lung Cancer Research. The article has undergone external 
peer review.

Reporting Checklist: The authors have completed the Narrative Review reporting checklist. Available at http:// dx.doi.org/10.21037/tlcr-20-806

Peer Review File: Available at http://dx.doi.org/10.21037/ tlcr-20-806

Conflicts of Interest: All authors have completed the ICMJE uniform disclosure form (available at http://dx.doi. org/10.21037/tlcr-20-806). The series "Lung cancer and the immune system" was commissioned by the editorial office without any funding or sponsorship. The authors have no other conflicts of interest to declare.

Ethical Statement: The authors are accountable for all aspects of the work in ensuring that questions related to the accuracy or integrity of any part of the work are appropriately investigated and resolved.

Open Access Statement: This is an Open Access article distributed in accordance with the Creative Commons Attribution-NonCommercial-NoDerivs 4.0 International License (CC BY-NC-ND 4.0), which permits the noncommercial replication and distribution of the article with the strict proviso that no changes or edits are made and the original work is properly cited (including links to both the formal publication through the relevant DOI and the license). See: https://creativecommons.org/licenses/by-nc-nd/4.0/.

\section{References}

1. Hodi FS, O'Day SJ, McDermott DF, et al. Improved survival with ipilimumab in patients with metastatic melanoma. N Engl J Med 2010;363:711-23.

2. Garon EB, Rizvi NA, Hui R, et al. Pembrolizumab for the treatment of non-small-cell lung cancer. N Engl J Med 2015;372:2018-28.

3. Brahmer J, Reckamp KL, Baas P, et al. Nivolumab versus Docetaxel in Advanced Squamous-Cell Non-Small-Cell Lung Cancer. N Engl J Med 2015;373:123-35.

4. Antonia SJ, Villegas A, Daniel D, et al. Overall Survival with Durvalumab after Chemoradiotherapy in Stage III NSCLC. N Engl J Med 2018;379:2342-50.

5. Forde PM, Chaft JE, Smith KN, et al. Neoadjuvant PD-1 Blockade in Resectable Lung Cancer. N Engl J Med 2018;378:1976-86.
6. DeWeerdt S. Bacteriology: a caring culture. Nature 2013;504:S4-5.

7. Pardoll DM. The blockade of immune checkpoints in cancer immunotherapy. Nat Rev Cancer 2012;12:252-64.

8. Ishida $\mathrm{Y}$, Agata $\mathrm{Y}$, Shibahara $\mathrm{K}$, et al. Induced expression of PD-1, a novel member of the immunoglobulin gene superfamily, upon programmed cell death. EMBO J 1992;11:3887-95.

9. Leach DR, Krummel MF, Allison JP. Enhancement of antitumor immunity by CTLA-4 blockade. Science 1996;271:1734-6.

10. Brahmer JR, Tykodi SS, Chow LQ, et al. Safety and activity of anti-PD-L1 antibody in patients with advanced cancer. N Engl J Med 2012;366:2455-65.

11. Barlesi F, Vansteenkiste J, Spigel D, et al. Avelumab versus docetaxel in patients with platinum-treated advanced non-small-cell lung cancer (JAVELIN Lung 200): an open-label, randomised, phase 3 study. Lancet Oncol 2018;19:1468-79.

12. Rittmeyer A, Barlesi F, Waterkamp D, et al. Atezolizumab versus docetaxel in patients with previously treated non-small-cell lung cancer (OAK): a phase 3, openlabel, multicentre randomised controlled trial. Lancet 2017;389:255-65.

13. Eggermont AM, Chiarion-Sileni V, Grob JJ, et al. Adjuvant ipilimumab versus placebo after complete resection of high-risk stage III melanoma (EORTC 18071): a randomised, double-blind, phase 3 trial. Lancet Oncol 2015;16:522-30.

14. Postow MA, Sidlow R, Hellmann MD. Immune-Related Adverse Events Associated with Immune Checkpoint Blockade. N Engl J Med 2018;378:158-68.

15. Buchbinder EI, Desai A. CTLA-4 and PD-1 Pathways: Similarities, Differences, and Implications of Their Inhibition. Am J Clin Oncol 2016;39:98-106.

16. Wei SC, Anang NAS, Sharma R, et al. Combination antiCTLA-4 plus anti-PD-1 checkpoint blockade utilizes cellular mechanisms partially distinct from monotherapies. Proc Natl Acad Sci U S A 2019;116:22699-709.

17. Chan KK, Bass AR. Autoimmune complications of immunotherapy: pathophysiology and management. BMJ 2020;369:m736.

18. Nishino M, Giobbie-Hurder A, Hatabu H, et al. Incidence of Programmed Cell Death 1 InhibitorRelated Pneumonitis in Patients With Advanced Cancer: A Systematic Review and Meta-analysis. JAMA Oncol 2016;2:1607-16.

19. Sears CR, Peikert T, Possick JD, et al. Knowledge Gaps 
and Research Priorities in Immune Checkpoint Inhibitorrelated Pneumonitis. An Official American Thoracic Society Research Statement. Am J Respir Crit Care Med 2019;200:e31-43.

20. Delaunay M, Cadranel J, Lusque A, et al. Immunecheckpoint inhibitors associated with interstitial lung disease in cancer patients. Eur Respir J 2017;50:1700050.

21. Leroy V, Templier C, Faivre JB, et al. Pembrolizumabinduced pneumonitis. ERJ Open Res 2017;3:00081-2016.

22. Wang H, Guo X, Zhou J, et al. Clinical diagnosis and treatment of immune checkpoint inhibitor-associated pneumonitis. Thorac Cancer 2020;11:191-7.

23. Suresh K, Naidoo J, Zhong Q, et al. The alveolar immune cell landscape is dysregulated in checkpoint inhibitor pneumonitis. J Clin Invest 2019;129:4305-15.

24. Ceschi A, Noseda R, Palin K, et al. Immune Checkpoint Inhibitor-Related Cytokine Release Syndrome: Analysis of WHO Global Pharmacovigilance Database. Front Pharmacol 2020;11:557.

25. Brudno JN, Kochenderfer JN. Toxicities of chimeric antigen receptor $\mathrm{T}$ cells: recognition and management. Blood 2016;127:3321-30.

26. Lim SY, Lee JH, Gide TN, et al. Circulating Cytokines Predict Immune-Related Toxicity in Melanoma Patients Receiving Anti-PD-1-Based Immunotherapy. Clin Cancer Res 2019;25:1557-63.

27. Som A, Mandaliya R, Alsaadi D, et al. Immune checkpoint inhibitor-induced colitis: A comprehensive review. World J Clin Cases 2019;7:405-18.

28. Naqash AR, Yang LV, Sanderlin EJ, et al. Interleukin-6 as one of the potential mediators of immune-related adverse events in non-small cell lung cancer patients treated with immune checkpoint blockade: evidence from a case report. Acta Oncol 2018;57:705-8.

29. Das R, Bar N, Ferreira M, et al. Early B cell changes predict autoimmunity following combination immune checkpoint blockade. J Clin Invest 2018;128:715-20.

30. Brahmer JR. PD-1-targeted immunotherapy: recent clinical findings. Clin Adv Hematol Oncol 2012;10:674-5.

31. Nishino M, Sholl LM, Hodi FS, et al. Anti-PD-1-Related Pneumonitis during Cancer Immunotherapy. N Engl J Med 2015;373:288-90.

32. Abdel-Rahman O, Fouad M. Risk of pneumonitis in cancer patients treated with immune checkpoint inhibitors: a meta-analysis. Ther Adv Respir Dis 2016;10:183-93.

33. Su Q, Zhu EC, Wu JB, et al. Risk of Pneumonitis and Pneumonia Associated With Immune Checkpoint Inhibitors for Solid Tumors: A Systematic Review and
Meta-Analysis. Front Immunol 2019;10:108.

34. Larkin J, Chiarion-Sileni V, Gonzalez R, et al. Combined Nivolumab and Ipilimumab or Monotherapy in Untreated Melanoma. N Engl J Med 2015;373:23-34.

35. Postow MA, Chesney J, Pavlick AC, et al. Nivolumab and ipilimumab versus ipilimumab in untreated melanoma. N Engl J Med 2015;372:2006-17.

36. Hellmann MD, Paz-Ares L, Bernabe Caro R, et al. Nivolumab plus Ipilimumab in Advanced Non-Small-Cell Lung Cancer. N Engl J Med 2019;381:2020-31.

37. Suresh K, Voong KR, Shankar B, et al. Pneumonitis in Non-Small Cell Lung Cancer Patients Receiving Immune Checkpoint Immunotherapy: Incidence and Risk Factors. J Thorac Oncol 2018;13:1930-9.

38. Cathcart-Rake EJ, Sangaralingham LR, Henk HJ, et al. A Population-based Study of Immunotherapy-related Toxicities in Lung Cancer. Clin Lung Cancer 2020;21:421427.e2.

39. Shimokawaji T, Narita S, Naito T, et al. Clinical characteristics of nivolumab-induced radiation recall pneumonitis in patients with non-small cell lung cancer: A multicenter real-world analysis of 669 patients. J Clin Oncol 2020;38:88.

40. Pasello G, Pavan A, Attili I, et al. Real world data in the era of Immune Checkpoint Inhibitors (ICIs): Increasing evidence and future applications in lung cancer. Cancer Treat Rev 2020;87:102031.

41. Wang DY, Salem JE, Cohen JV, et al. Fatal Toxic Effects Associated With Immune Checkpoint Inhibitors: A Systematic Review and Meta-analysis. JAMA Oncol 2018;4:1721-8.

42. Moey MYY, Gougis P, Goldschmidt V, et al. Increased reporting of fatal pneumonitis associated with immune checkpoint inhibitors: a WHO pharmacovigilance database analysis. Eur Respir J 2020;55:2000038.

43. Shohdy KS, Abdel-Rahman O. Risk of pneumonitis with different immune checkpoint inhibitors in NSCLC. Ann Transl Med 2017;5:365.

44. Pillai RN, Behera M, Owonikoko TK, et al. Comparison of the toxicity profile of PD-1 versus PD-L1 inhibitors in non-small cell lung cancer: A systematic analysis of the literature. Cancer 2018;124:271-7.

45. Cui P, Liu Z, Wang G, et al. Risk factors for pneumonitis in patients treated with anti-programmed death-1 therapy: A case-control study. Cancer Med 2018;7:4115-20.

46. Suzuki Y, Karayama M, Uto T, et al. Assessment of Immune-Related Interstitial Lung Disease in Patients With NSCLC Treated with Immune Checkpoint 
Inhibitors: A Multicenter Prospective Study. J Thorac Oncol 2020;15:1317-27.

47. Cho JY, Kim J, Lee JS, et al. Characteristics, incidence, and risk factors of immune checkpoint inhibitor-related pneumonitis in patients with non-small cell lung cancer. Lung Cancer 2018;125:150-6.

48. Kanai O, Kim YH, Demura Y, et al. Efficacy and safety of nivolumab in non-small cell lung cancer with preexisting interstitial lung disease. Thorac Cancer 2018;9:847-55.

49. Fujimoto D, Yomota M, Sekine A, et al. Nivolumab for advanced non-small cell lung cancer patients with mild idiopathic interstitial pneumonia: A multicenter, open-label single-arm phase II trial. Lung Cancer 2019;134:274-8.

50. Shaverdian N, Lisberg AE, Bornazyan K, et al. Previous radiotherapy and the clinical activity and toxicity of pembrolizumab in the treatment of non-small-cell lung cancer: a secondary analysis of the KEYNOTE-001 phase 1 trial. Lancet Oncol 2017;18:895-903.

51. Antonia SJ, Villegas A, Daniel D, et al. Durvalumab after Chemoradiotherapy in Stage III Non-Small-Cell Lung Cancer. N Engl J Med 2017;377:1919-29.

52. Avrillon V, Bota Ouchlif S, Merle P, et al. 1470P - First real life data on durvalumab after definitive concomitant chemoradiotherapy (cCRT) in unresectable stage (St) III non-small cell lung cancer (NSCLC) in France: Analysis of 591 patients (pts) enrolled in the French cohort (c) temporary authorization of use (ATU). Ann Oncol 2019;30:v597.

53. Miura Y, Mouri A, Kaira K, et al. Chemoradiotherapy followed by durvalumab in patients with unresectable advanced non-small cell lung cancer: Management of adverse events. Thorac Cancer 2020;11:1280-7.

54. Jung HA, Noh JM, Sun JM, et al. Real world data of durvalumab consolidation after chemoradiotherapy in stage III non-small-cell lung cancer. Lung Cancer 2020;146:23-9.

55. Vansteenkiste J, Naidoo J, Faivre-Finn C, et al. MA05.02 PACIFIC Subgroup Analysis: Pneumonitis in Stage III, Unresectable NSCLC Patients Treated with Durvalumab vs. Placebo After CRT. J Thorac Oncol 2018;13:S370-1.

56. Togashi Y, Masago K, Mishima M, et al. A case of radiation recall pneumonitis induced by erlotinib, which can be related to high plasma concentration. J Thorac Oncol 2010;5:924-5.

57. Schweitzer VG, Juillard GJ, Bajada CL, et al. Radiation recall dermatitis and pneumonitis in a patient treated with paclitaxel. Cancer 1995;76:1069-72.
58. Louvel G, Bahleda R, Ammari S, et al. Immunotherapy and pulmonary toxicities: can concomitant immunecheckpoint inhibitors with radiotherapy increase the risk of radiation pneumonitis? Eur Respir J 2018;51:1701737.

59. Tamiya A, Tamiya M, Nakahama K, et al. Correlation of Radiation Pneumonitis History Before Nivolumab with Onset of Interstitial Lung Disease and Progression-free Survival of Patients with Pre-treated Advanced Non-small Cell Lung Cancer. Anticancer Res 2017;37:5199-205.

60. Klein SL, Flanagan KL. Sex differences in immune responses. Nat Rev Immunol 2016;16:626-38.

61. Conforti F, Pala L, Bagnardi V, et al. Cancer immunotherapy efficacy and patients' sex: a systematic review and meta-analysis. Lancet Oncol 2018;19:737-46.

62. Abdel-Rahman O. Does a patient's sex predict the efficacy of cancer immunotherapy? Lancet Oncol 2018;19:716-7.

63. Duma N, Abdel-Ghani A, Yadav S, et al. Sex Differences in Tolerability to Anti-Programmed Cell Death Protein 1 Therapy in Patients with Metastatic Melanoma and NonSmall Cell Lung Cancer: Are We All Equal? Oncologist 2019;24:e1148-55.

64. Naidoo J, Wang X, Woo KM, et al. Pneumonitis in Patients Treated With Anti-Programmed Death-1/ Programmed Death Ligand 1 Therapy. J Clin Oncol 2017;35:709-17.

65. Tirumani SH, Ramaiya NH, Keraliya A, et al. Radiographic Profiling of Immune-Related Adverse Events in Advanced Melanoma Patients Treated with Ipilimumab. Cancer Immunol Res 2015;3:1185-92.

66. Wang GX, Guo LQ, Gainor JF, et al. Immune Checkpoint Inhibitors in Lung Cancer: Imaging Considerations. AJR Am J Roentgenol 2017;209:567-75.

67. Johnson DB, Balko JM, Compton ML, et al. Fulminant Myocarditis with Combination Immune Checkpoint Blockade. N Engl J Med 2016;375:1749-55.

68. Safa H, Johnson DH, Trinh VA, et al. Immune checkpoint inhibitor related myasthenia gravis: single center experience and systematic review of the literature. J Immunother Cancer 2019;7:319.

69. Berthod G, Lazor R, Letovanec I, et al. Pulmonary sarcoid-like granulomatosis induced by ipilimumab. J Clin Oncol 2012;30:e156-9.

70. Fujita K, Terashima T, Mio T. Anti-PD-1 Antibody Treatment and the Development of Acute Pulmonary Tuberculosis. J Thorac Oncol 2016;11:2238-40.

71. Blanchard A, Bouchard N. Pembrolizumab-induced obstructive bronchiolitis in a patient with stage IV nonsmall-cell lung cancer. Curr Oncol 2019;26:e571-3. 
72. Mitropoulou G, Daccord C, Sauty A, et al. Immunotherapy-Induced Airway Disease: A New Pattern of Lung Toxicity of Immune Checkpoint Inhibitors. Respiration 2020;99:181-6.

73. Balagani A, Arain MH, Sheshadri A. Bronchiolitis Obliterans after Combination Immunotherapy with Pembrolizumab and Ipilimumab. Journal of Immunotherapy and Precision Oncology 2020;1:49-52.

74. Baba T, Sakai F, Kato T, et al. Radiologic features of pneumonitis associated with nivolumab in non-smallcell lung cancer and malignant melanoma. Future Oncol 2019;15:1911-20.

75. Watanabe S, Ota T, Hayashi M, et al. Prognostic significance of the radiologic features of pneumonitis induced by anti-PD-1 therapy. Cancer Med 2020;9:3070-7.

76. Travis WD, Costabel U, Hansell DM, et al. An official American Thoracic Society/European Respiratory Society statement: Update of the international multidisciplinary classification of the idiopathic interstitial pneumonias. Am J Respir Crit Care Med 2013;188:733-48.

77. Nishino M, Ramaiya NH, Awad MM, et al. PD-1 Inhibitor-Related Pneumonitis in Advanced Cancer Patients: Radiographic Patterns and Clinical Course. Clin Cancer Res 2016;22:6051-60.

78. Rashdan S, Minna JD, Gerber DE. Diagnosis and management of pulmonary toxicity associated with cancer immunotherapy. Lancet Respir Med 2018;6:472-8.

79. Brahmer JR, Lacchetti C, Schneider BJ, et al. Management of Immune-Related Adverse Events in Patients Treated With Immune Checkpoint Inhibitor Therapy: American Society of Clinical Oncology Clinical Practice Guideline. J Clin Oncol 2018;36:1714-68.

80. Schwaiblmair M, Behr W, Haeckel T, et al. Drug induced interstitial lung disease. Open Respir Med J 2012;6:63-74.

81. Flieder DB, Travis WD. Pathologic characteristics of druginduced lung disease. Clin Chest Med 2004;25:37-45.

82. Haanen J, Carbonnel F, Robert C, et al. Management of toxicities from immunotherapy: ESMO Clinical Practice Guidelines for diagnosis, treatment and follow-up. Ann Oncol 2017;28:iv119-42.

83. Koelzer VH, Rothschild SI, Zihler D, et al. Systemic inflammation in a melanoma patient treated with immune checkpoint inhibitors-an autopsy study. J Immunother Cancer 2016;4:13.

84. Shea M, Rangachari D, Hallowell RW, et al. Radiologic and autopsy findings in a case of fatal immune checkpoint inhibitor-associated pneumonitis. Cancer Treat Res
Commun 2018;15:17-20.

85. Larsen BT, Chae JM, Dixit AS, et al. Clinical and Histopathologic Features of Immune Checkpoint Inhibitor-related Pneumonitis. Am J Surg Pathol 2019;43:1331-40.

86. Bui A, Han S, Alexander M, et al. Pulmonary function testing for the early detection of drug induced lung disease: a systematic review in adults treated with drugs associated with pulmonary toxicity. Intern Med J 2020;50:1311-25.

87. Franzen D, Schad K, Kowalski B, et al. Ipilimumab and early signs of pulmonary toxicity in patients with metastastic melanoma: a prospective observational study. Cancer Immunol Immunother 2018;67:127-34.

88. Colen RR, Fujii T, Bilen MA, et al. Radiomics to predict immunotherapy-induced pneumonitis: proof of concept. Invest New Drugs 2018;36:601-7.

89. Majem M, Garcia-Martinez E, Martinez M, et al. SEOM clinical guideline for the management of immune-related adverse events in patients treated with immune checkpoint inhibitors (2019). Clin Transl Oncol 2020;22:213-22.

90. Puzanov I, Diab A, Abdallah K, et al. Managing toxicities associated with immune checkpoint inhibitors: consensus recommendations from the Society for Immunotherapy of Cancer (SITC) Toxicity Management Working Group. J Immunother Cancer 2017;5:95.

91. Thompson JA, Schneider BJ, Brahmer J, et al. Management of Immunotherapy-Related Toxicities, Version 1.2019. J Natl Compr Canc Netw 2019;17:255-89.

92. Sawai Y, Katsuya Y, Shinozaki-Ushiku A, et al. Rapid temporal improvement of pembrolizumab-induced pneumonitis using the anti-TNF-alpha antibody infliximab. Drug Discov Ther 2019;13:164-7.

93. Lai K, Shannon VR, Balachandran D, et al. Role of Infliximab in Immune Check Point Inhibitor Induced Pneumonitis. C38. AUTOIMMUNE, DRUG RELATED, AND TRANSPLANT: American Thoracic Society; 2019. p. A4736-A.

94. Cooksley T, Marshall W, Gupta A. Early infliximab in life-threatening immune-mediated pneumonitis. QJM 2019;112:929-30.

95. Johnson DH, Zobniw CM, Trinh VA, et al. Infliximab associated with faster symptom resolution compared with corticosteroids alone for the management of immunerelated enterocolitis. J Immunother Cancer 2018;6:103.

96. Pollack MH, Betof A, Dearden H, et al. Safety of resuming anti-PD-1 in patients with immune-related adverse events (irAEs) during combined anti-CTLA-4 and anti-PD-1 in 
metastatic melanoma. Ann Oncol 2018;29:250-5.

97. de Jong C, Peters BJM, Schramel F. Recurrent

Episodes of Nivolumab-Induced Pneumonitis after

Nivolumab Discontinuation and the Time Course of

Carcinoembryonic Antigen Levels: A Case of a 58-Year-

Old Woman with Non-Small Cell Lung Cancer.

Chemotherapy 2018;63:272-7.

98. Bronstein Y, Ng CS, Hwu P, et al. Radiologic

Cite this article as: Miller AR, Manser R. The knowns \& unknowns of pulmonary toxicity following immune checkpoint inhibitor therapies: a narrative review. Transl Lung Cancer Res 2021;10(6):2752-2765. doi: 10.21037/tlcr-20-806 manifestations of immune-related adverse events in patients with metastatic melanoma undergoing antiCTLA-4 antibody therapy. AJR Am J Roentgenol 2011;197:W992-1000.

99. Weber JS, Hodi FS, Wolchok JD, et al. Safety Profile of Nivolumab Monotherapy: A Pooled Analysis of Patients With Advanced Melanoma. J Clin Oncol 2017;35:785-92. 